\section{$1 \mathrm{P549}$}

Both MHC class II and its GPI-anchored form undergo hop diffusion as observed by single-molecule tracking

Yasuhiro Umemura ${ }^{1}$, Takahiro Fujiwara ${ }^{1}$, Kenichi Suzuki ${ }^{1}$, Marija $\mathrm{Vrljic}^{2}$, Stefanie Y. Nishimura ${ }^{2}$, W. E. Moerner ${ }^{2}$, Harden M. McConnell ${ }^{2}$, Akihiro Kusumi ${ }^{1}$ ${ }^{1}$ Membrane Mechanisms Project, ICORP-IST, Inst. for Frontier Med. Sci., Kyoto Univ., ${ }^{2}$ Dept. of Chem. Stanford Univ.

Fujiwara et al. (2002) showed that the entire cell membrane (except for caveolae, clathrin-coated pits, and cell adhesion structures) is partitioned into many small compartments of $30-200 \mathrm{~nm}$, due to the presence of the actin-based MSK and various transmembrane proteins anchored to the MSK, which form the compartment boundaries. In short-term, phospholipids are confined within a compartment and in long-term, they undergo hop movements between adjacent compartments (hop diffusion). Such partitioning of the cell membrane and hop diffusion of phospholipids were found in all of the eight cultured cell lines we examined thus far (Murase et al., 2004). On the other hand, single-fluorescentmolecule tracking at a 100-ms resolution by Vrljic et al. (2002) found that the transmembrane MHC Class II protein and its GPI-anchored modified form expressed in CHO cells undergo Brownian diffusion without any influence of actin depolymerization, at apparent variance with our observations. Here, we addressed this issue using the same molecules expressed in the same cell line, using highspeed single-molecule tracking. At a $20-\mu$ s resolution, both molecules exhibited rapid hop diffusion between $\sim 40-\mathrm{nm}$ compartments with a dwell time of 1-2 ms in each compartment on average. Partial actin depolymerization increased the compartment size with concomitant decreases of the hop rates, which explains why the macroscopic diffusion rate observed at a 33-ms resolution hardly changed after actin depolymerization. These results support fence and picket models.

\section{$1 P 551$}

Single hydrogen bonds of DNA base pairs detected by intermolecular force microscopy

OMichio Hiroshima ${ }^{1,2}$, Makio Tokunaga ${ }^{1,2,3}$

'RCAI, RIKEN, ${ }^{2}$ Natl. Inst. of Genetics, ${ }^{3}$ Grad. Univ. for Adv. Studies

Single hydrogen bonds of DNA base pairs have been resolved by unzipping double-stranded DNA molecules with intermolecular force microscope (IFM), as we have already reported. To detect such ultrafine forces, high force resolution as well as high accuracy in probe positioning is required. Subpiconewton resolution was achieved using ultra-sensitive cantilevers. The probe position is controlled with nanometer accuracy using laser radiation pressure with feedback.

In the recent study, we refined force-extension curves by averaging data obtained in further measurements. A control experiment using another DNA molecule which contains only a few base pairs was also carried out.

Force-extension curves showed repeated force peaks of $10-20 \mathrm{pN}$. The previous studies showed that the force for separating poly $(\mathrm{G}-\mathrm{C})$ DNA was 1.5 to 2 times stronger than that for poly(A-T). However, we found no such difference in the force between individual G-C and A-T base pairs.

The force-extension curves are averaged over sequence repeats using correlation functions. The force curve of individual G-C and A-T pairs is composed of three and two peaks, respectively, which are assigned to single hydrogen bonds.

The force is variable depending on the distuption length but the work is constant. The work to disrupt single hydrogen bonds is thermal energy level. This is the first report to detect directly the force of single hydrogen bonds in biological macromolecules.

\section{$1 \mathrm{P550}$}

Detection of transient arrest of lateral diffusion of membrane molecules in single-molecule tracking trajectories

OKuniko Sakamoto, Takahiro Fujiwara, Akihiro Kusumi

Membrane Mechanisms Project, ICORP-JST, Kyoto Univ.

As investigations by single-molecule tracking are advanced, many investigators realized that many signaling molecules in/on the plasma membrane undergo Transient Arrest of LateraL diffusion (TALL), when the cells are stimulated. However, many single-molecule trajectories are as short as only 10-30 frames (0.33-1.00 second). Furthermore, since the $\mathrm{S} / \mathrm{N}$ ratio of the single-molecule image is not high, the precision for determining the positions (coordinates) of single molecules in the image is low. Therefore, it is becoming clear that a statistical method has to be developed to detect TALLs at a known probability under the given trajectory length and the $\mathrm{S} / \mathrm{N}$ of the fluorescent spot, which would allow us to determine if the observed apparent changes in frequency of TALL after cell stimulation are meaningful. Here, we developed a method to detect TALL in a Brownian trajectory using the mean-square displacements calculated for systematically varied window sizes assigned to each point in the trajectory. By applying this analysis to two kinds of simulated trajectories that include random. errors of position determination, one based on simple Brownian diffusion and the other which includes TALL in Brownian motion, we found several useful parameters that are necessary for TALL detection as well as for TALL characterization. This software was successfully tested for experimental trajectories that appeared to include TALL.

\section{$1 \mathrm{P552}$}

\author{
Snapshots of DNA untangling by a type-II topoisomerase \\ OKatsunori Yogo, Kazuhiko Kinosita \\ Waseda Univ.
}

Type-II topoisomerases are ubiquitous ATP-dependent enzymes that play key roles in the maintenance of DNA topology in cells. They control the supercoiling of DNA and untangle the catenanes that arise during replication or recombination. Lack of their activities during cell division ultimately causes cell death.Like a magician who fascinates the audience by solving a knot of rope without touching the knot, topo II untangles DNA catenanes by catalyzing the transport of one DNA segment through a transient double-stranded break in a second. We aim at showing this unlinking magic directly under a microscope and revealing the functional mechanism in detail. 